\title{
Galileons, phantom fields, and the fate of the Universe
}

\author{
M. Shahalam ${ }^{1, a}$, S. K. J. Pacif ${ }^{2, b}$, R. Myrzakulov ${ }^{3, c}$ \\ ${ }^{1}$ Department of Physics, Aligarh Muslim University, Aligarh, India \\ 2 Center For Theoretical Physics, Jamia Millia Islamia, New Delhi, India \\ ${ }^{3}$ Department of General and Theoretical Physics, Eurasian National University, Astana, Kazakhstan
}

Received: 27 April 2016 / Accepted: 6 July 2016 / Published online: 21 July 2016

(c) The Author(s) 2016. This article is published with open access at Springerlink.com

\begin{abstract}
In this paper we study cosmological dynamics of the phantom as well as non-phantom fields with a linear potential in the presence of a Galileon correction $\left(\partial_{\mu} \phi \partial^{\mu} \phi\right) \square \phi$. We show that the Big Crunch singularity is delayed compared to the standard case; the delay crucially depends upon the strength of a Galileon correction. As for the phantom Galileon, $\rho_{\phi}$ is shown to grow more slowly compared to the standard phantom delaying the approach of the singularity. In the case, $V \sim \phi^{n}, n>4$, Big Rip is also delayed, similar phenomenon is shown to take place for potentials steeper than the exponential.
\end{abstract}

\section{Introduction}

Observations in cosmology have recently led to the confirmation that the Universe is undergoing an accelerated phase of the expansion at present [1-5]. The direct support for the phenomenon came from the observations of supernovae of type Ia (SNe Ia) [1]. The explosions of these SNe Ia look fainter than expected in the Einstein-de Sitter model. The concept of "dark energy" was introduced to explain the luminosityredshift observations of these type Ia supernovae by modifying the right hand side of Einstein field equations, which gives rise to an accelerated expansion of the Universe and thus explains the unexpected faintness of the supernovae. The weird form of energy yet remains mysterious as there is no direct observational test to probe it but it is generally assumed that it has a large negative pressure $[6,7]$.

In the past few years there have been a number of activities for modelling dark energy including the models with the scalar field and brane world etc. To this effect, a large variety of scalar field models are discussed in the literature including quintessence [8-14], K-essence [15,16], spintessence [17],

\footnotetext{
a e-mail: mohdshahamu@gmail.com

b e-mail: shibesh.math@gmail.com

c e-mail: rmyrzakulov@gmail.com
}

tachyon [18,19], quintom [20-27], chameleon [28-31] and many more. These models of the scalar field give the equation of state parameter $w \geq-1$. It is interesting to note that the observational data also allows models of dark energy with equation of state parameter crossing the -1 line (called phantom field models). Thus, a number of the phantom models have been discussed in the literature [32-45], for instance, brane world and non-minimally coupled scalar field models can give phantom energy [46-49]. The simplest way to introduce the phantom effect is provided by a scalar field with a negative kinetic energy term which could be motivated from S-brane constructs in string theory [50-58]. The concept of the phantom field was first used in the steady state theory of Hoyle and subsequently incorporated in the Hoyle and Narlikar theory of gravitation [59-61].

The future singularity termed "Big Rip" [62] naturally arises in models with $w<-1$ and is characterised by the divergence of the scale factor after a finite interval of time. It is generic to keep $w$ time dependent rather than to consider it as a constant. This choice of $w$ generates specific scalar field models to avoid the cosmic doomsday [42,63-65] which requires a particular class of the phantom field potentials.

There are alternative ways to explain the accelerated expansion by modifying the left hand side of the Einstein field equations à la modified theories of gravity. Following this, a special class of dark energy models based on the large scale modification of gravity called Galileon gravity [6679] was proposed. The distinguishing feature of this theory is that it provides a consistent modification of general relativity leaving the local physics intact. This modified gravity in this scheme can give rise to the observed late time cosmic acceleration and also it is free from negative energy instabilities. The Galileon field has five field Lagrangians $\mathcal{L}_{i}$ $(i=1, \ldots, 5)$ in 4-dimensional space-time. The Lagrangian $\mathcal{L}_{1}$ is linear, $\mathcal{L}_{2}$ is the standard kinetic term and $\mathcal{L}_{3}$ represents the Vainshtein term, consisting of three Galileon fields, that is related to the decoupling limit of Dvali, Gabadadze, and 
Porrati model [80,81], while $\mathcal{L}_{4}$ and $\mathcal{L}_{5}$ consist of higher order non-linear derivative terms of the field. In the case we study the scalar field with linear potential, it becomes obligatory to complement it by the higher derivative Galileon terms in the Lagrangian. For simplicity, we shall consider the lowest Galileon term $\mathcal{L}_{3}$ for phantom and non-phantom fields with a linear potential. On purely phenomenological grounds, we also examine the phantom case with a general potential term $V(\phi)$ [82-84] complemented by a Galileon term. In this case, we focus on some general features of the cosmological dynamics, in particular, current acceleration and future evolution of the Universe.

Recently, it was found that in quintessence models where scalar field potentials become negative might lead to a collapse of the Universe in the distant future [85-89], dubbed the Big Crunch singularity. Lykkas and Perivolaropoulos show that the cosmic doomsday singularity can be avoided in scalar-tensor quintessence with a linear potential by some values of the non-minimal coupling parameter [90]. In this paper, we shall examine these and other aforesaid issues in the presence of a Galileon correction $\mathcal{L}_{3}$ in the Lagrangian.

The paper is organised as follows. In Sect. 2, we consider a Galileon field model with a linear potential which is generically non-minimally coupled scalar field model and investigate the present and future evolution of the Universe for both the phantom and the non-phantom cases. In Sect. 3, we consider a Galileon phantom field model with steep exponential potential and examine the future evolution of the Universe. We summarise our results in Sect. 4.

\section{Galileon field with a linear potential}

In this section, we consider the Galileon field action possessing up to the third order term in the Lagrangian with a field potential $V(\phi)$ [82-84],

$$
\begin{aligned}
S & =\int \mathrm{d}^{4} x \sqrt{-g} \\
& \times\left[\frac{M_{p l}^{2}}{2} R-\frac{1}{2} \epsilon(\nabla \phi)^{2}-\frac{\beta}{2 M^{3}}(\nabla \phi)^{2} \square \phi-V(\phi)\right]+S_{m}
\end{aligned}
$$

where $\epsilon=-1$ and +1 , for phantom and non-phantom Galileon fields, respectively. $M_{p l}^{2}=1 / 8 \pi G$ is the reduced Planck mass and the constant $\beta$ is dimensionless. $S_{m}$ is for the matter action and $M$ is a constant of mass dimension one. For simplicity, we fix here $M=M_{p l}$. In a homogeneous isotropic flat Friedmann-Lemaitre-Robertson-Walker (FLRW) Universe, the equations of motion are obtained by varying the action (1) with respect to the metric tensor $g_{\mu \nu}$ and the scalar field $\phi$ as

$$
\begin{aligned}
& 3 M_{p l}^{2} H^{2}=\rho_{m}+\frac{1}{2} \epsilon \dot{\phi}^{2}-3 \frac{\beta}{M_{\mathrm{pl}}^{3}} H \dot{\phi}^{3}+V(\phi), \\
& M_{p l}^{2}\left(2 \dot{H}+3 H^{2}\right)=-\frac{1}{2} \epsilon \dot{\phi}^{2}-\frac{\beta}{M_{\mathrm{pl}}^{3}} \dot{\phi}^{2} \ddot{\phi}+V(\phi), \\
& \frac{\ddot{a}}{a}=-\frac{1}{6 M_{p l}^{2}}\left(\rho_{m}+2 \epsilon \dot{\phi}^{2}-\frac{3 \beta}{M_{p l}^{3}}\left(H \dot{\phi}^{3}-\dot{\phi}^{2} \ddot{\phi}\right)-2 V(\phi)\right), \\
& \epsilon \ddot{\phi}+3 H \epsilon \dot{\phi}-3 \frac{\beta}{M_{\mathrm{pl}}^{3}} \dot{\phi}\left(3 H^{2} \dot{\phi}+\dot{H} \dot{\phi}+2 H \ddot{\phi}\right)+V^{\prime}(\phi)=0,
\end{aligned}
$$

where / denotes the derivative with respect to $\phi$ and $\rho_{m}$ is the energy density of matter. The energy conservation equation of matter is given by

$\dot{\rho}_{m}+3 H \rho_{m}=0$.

In the radiation/matter dominated phase, the Universe is dominated by a perfect fluid with equation of state $p=w \rho$. In this phase of evolution the density of matter $\rho_{m}$ dominates over the field $\phi$. With the expansion of the Universe over time the Hubble parameter $H$ begins to decrease and the scalar field $\phi$ starts to dominate. The total energy content of the Universe $\rho_{\text {total }}=\rho_{m}+\rho_{\phi} \simeq \rho_{\phi}=\frac{1}{2} \epsilon \dot{\phi}^{2}+V(\phi)-\frac{3 \beta}{M_{p l}^{3}} H \dot{\phi}^{3}$. Therefore Eq. (2) reduces to

$3 M_{p l}^{2} H^{2}=\frac{1}{2} \epsilon \dot{\phi}^{2}+V(\phi)-\frac{3 \beta}{M_{p l}^{3}} H \dot{\phi}^{3}$,

which is difficult to solve analytically. In the following, we shall solve the evolution equations numerically and plot the future evolution graphically.

We introduce the dimensionless parameters

$$
\begin{aligned}
& H_{0} t \longrightarrow t, \\
& \frac{\phi}{\sqrt{3} M_{p l}} \longrightarrow \phi, \\
& \frac{V_{0}}{\sqrt{3} M_{p l}^{2} H_{0}^{2}} \longrightarrow V_{0} .
\end{aligned}
$$

The system of equations (4) and (5) can be written as

$$
\begin{aligned}
\frac{\ddot{a}}{a}= & -\epsilon \dot{\phi}^{2}+\frac{3 \sqrt{3} \beta}{2}\left(\frac{H_{0}}{M_{p l}}\right)^{2}\left(\frac{\dot{a}}{a} \dot{\phi}^{3}-\dot{\phi}^{2} \ddot{\phi}\right) \\
& +V_{0} \phi-\frac{\Omega_{m 0}}{2 a^{3}}, \\
\epsilon \ddot{\phi} & +3 \frac{\dot{a}}{a} \epsilon \dot{\phi}-3 \sqrt{3} \beta \dot{\phi}\left(\frac{H_{0}}{M_{p l}}\right)^{2} \\
& \times\left\{3 \frac{\dot{a}^{2}}{a^{2}} \dot{\phi}+\left(\frac{a \ddot{a}-\dot{a}^{2}}{a^{2}}\right) \dot{\phi}+2 \frac{\dot{a}}{a} \ddot{\phi}\right\}+V_{0}=0 .
\end{aligned}
$$

The equation of state parameter $w$ for a Galileon field is defined as 


$$
\begin{aligned}
w & =\frac{p_{\phi}}{\rho_{\phi}} ; \\
p_{\phi} & =\frac{1}{2} \epsilon \dot{\phi}^{2}+\frac{\beta}{M_{\mathrm{pl}}^{3}} \dot{\phi}^{2} \ddot{\phi}-V(\phi), \\
\rho_{\phi} & =\frac{1}{2} \epsilon \dot{\phi}^{2}-3 \frac{\beta}{M_{\mathrm{pl}}^{3}} H \dot{\phi}^{3}+V(\phi) .
\end{aligned}
$$

In the case of the Galileon field model, we consider two cases, phantom and non-phantom. First we shall discuss the non-phantom case.

\section{Case I: Non-phantom $(\epsilon=+1)$}

When $\beta=0$ the Galileon field action (1) reduces to the standard quintessence field. In Eqs. (9) and (10), we have two variables, namely, scale factor $a$ and field $\phi$. The term $3 \beta H \dot{\phi}^{3} / M_{p l}^{3}$ in Eq. (2) is the Galileon correction term which depends upon $a, \phi$ and parameter $\beta$. The different values of $\beta$ puts the strength of a Galileon correction term over the quintessence term. For $\beta=0$, the evolution of the Galileon field model is the same as the standard quintessence throughout the history of the Universe. Hence all non-zero values of $\beta$ find the departure from quintessence and also the effect of Galileon correction term. Therefore in this analysis we take $\beta$ as a model parameter.

Now we solve Eqs. (9) and (10) numerically with the assumption that the field $\phi$ was frozen initially (i.e. $\phi\left(t_{i}\right)=$ $\phi_{i}$ and $\dot{\phi}\left(t_{i}\right)=0$ ) caused by huge Hubble damping. This is identical to thawing types of models [91-95]. We use the following initial conditions $\left(t \rightarrow t_{i} \simeq 0\right)$ :

$$
\begin{aligned}
a\left(t_{i}\right) & =\left(\frac{9 \Omega_{0 m}}{4}\right)^{1 / 3} t_{i}^{2 / 3}, \\
\dot{a}\left(t_{i}\right) & =\frac{2}{3}\left(\frac{9 \Omega_{0 m}}{4}\right)^{1 / 3} t_{i}^{-1 / 3} \\
\phi\left(t_{i}\right) & =\phi_{i} \\
\dot{\phi}\left(t_{i}\right) & =0 .
\end{aligned}
$$

With the above initial conditions and by tuning $\phi_{i}$, we get the following parameters at the present time:

$$
\begin{aligned}
& a\left(t_{0}\right)=1, \\
& H\left(t_{0}\right)=1, \\
& \Omega_{0 m}=0.3,
\end{aligned}
$$

where $t_{0}$ is defined as the time when the scale factor is unity. In the upper panels of Fig. 1, we present the dynamical evolution of field $\phi$ and scale factor $a$ for different values of $\beta$ and $V_{0}=1$. For $\beta=0$, the evolution of $a$ is alike to the standard quintessence model. Initially, the field is positive and the Universe gets expansion with the late time cosmic acceleration as soon as the field changes sign, in the future, the potential becomes negative and the scale factor collapses to a Big Crunch singularity. However, for larger values of $\beta$ the sign of the field changes in more distant future and correspondingly $V(\phi)$ becomes negative. Therefore, the collapse of scale factor is shifted into a more distant future for higher values of $\beta$. In other words, the cosmic doomsday is delayed for $\beta>0$.

In the lower left panel of Fig. 1, we show the evolution of the energy density for various values of $\beta$ and $V_{0}=1$. Initially, the Galileon field imitates a $\Lambda$ CDM like behaviour and its energy density is highly sub-dominant to the matter energy density $\rho_{m}$ and persists so, for most of the time of the expansion. The Galileon field remains in the state with $w=-1$ till the epoch $\rho_{\phi}$ comes close to $\rho_{m}$. At late times, the energy density of the Galileon field gets to the matter, overtakes it and begins decreasing $(w>-1)$, and the Universe acquires the present accelerated expansion having $\Omega_{0 m} \simeq 0.3$ and $\Omega_{0 \phi} \simeq 0.7$. Thereafter $\rho_{\phi}$ continuously decreases until it comes at a point where $\phi$ is negative (i.e. $\phi<0)$ and $\dot{\phi}^{2} / 2+V(\phi)-3 \beta H \dot{\phi}^{3} / M_{p l}^{3}=0$. Therefore, $H \longrightarrow 0$, i.e. the total energy density of the Universe reaches to zero and bounce occurs. For $\beta=0$, the Galileon field behaves as standard quintessence and a similar behaviour for the quintessence is shown in Ref. [89]. As we go for higher values of $\beta(\beta=0.3,0.5,1,10,100)$ the bounce and collapse get shifted into a distant future. One can say that the bounce and collapse are delayed for higher values of $\beta$.

The evolution of the equation of state for $V_{0}=1$ and various values of $\beta$ is shown in the lower right panel of Fig. 1 . For $\beta=0$, the equation of state of the Galileon field reduces to the equation of state of standard quintessence and diverges from the equation of state of $\Lambda \mathrm{CDM}$ model. As the values of $\beta$ are increased, we get more and more deviation in $w$ from the case of standard quintessence and the $\Lambda \mathrm{CDM}$ model is approached. The higher values of $\beta$ for the Galileon field with linear potential are in good agreement with the observations as shown in Ref. [83] where they have imposed observational constraints on the Galileon correction term which is associated with $\beta$.

\section{Case II: Phantom $(\epsilon=-1)$}

First we consider the case of $\beta=0$. Hence, the action (1) reduces to the action of the phantom field minimally coupled to gravity and matter,

$$
S=\int \mathrm{d}^{4} x \sqrt{-g}\left[\frac{M_{p l}^{2}}{2} R+\frac{1}{2}(\nabla \phi)^{2}-V(\phi)\right]+S_{m}
$$

The wrong sign in the kinetic energy term of Eq. (14) gives the ghost field in the context of quantum field theory or the phantom field in cosmology. As a dark energy candidate, the equation of state of the phantom field is marginally favoured by the present observations [96]. The vital cosmological dynamics of the phantom field has been broadly discussed in the liter- 

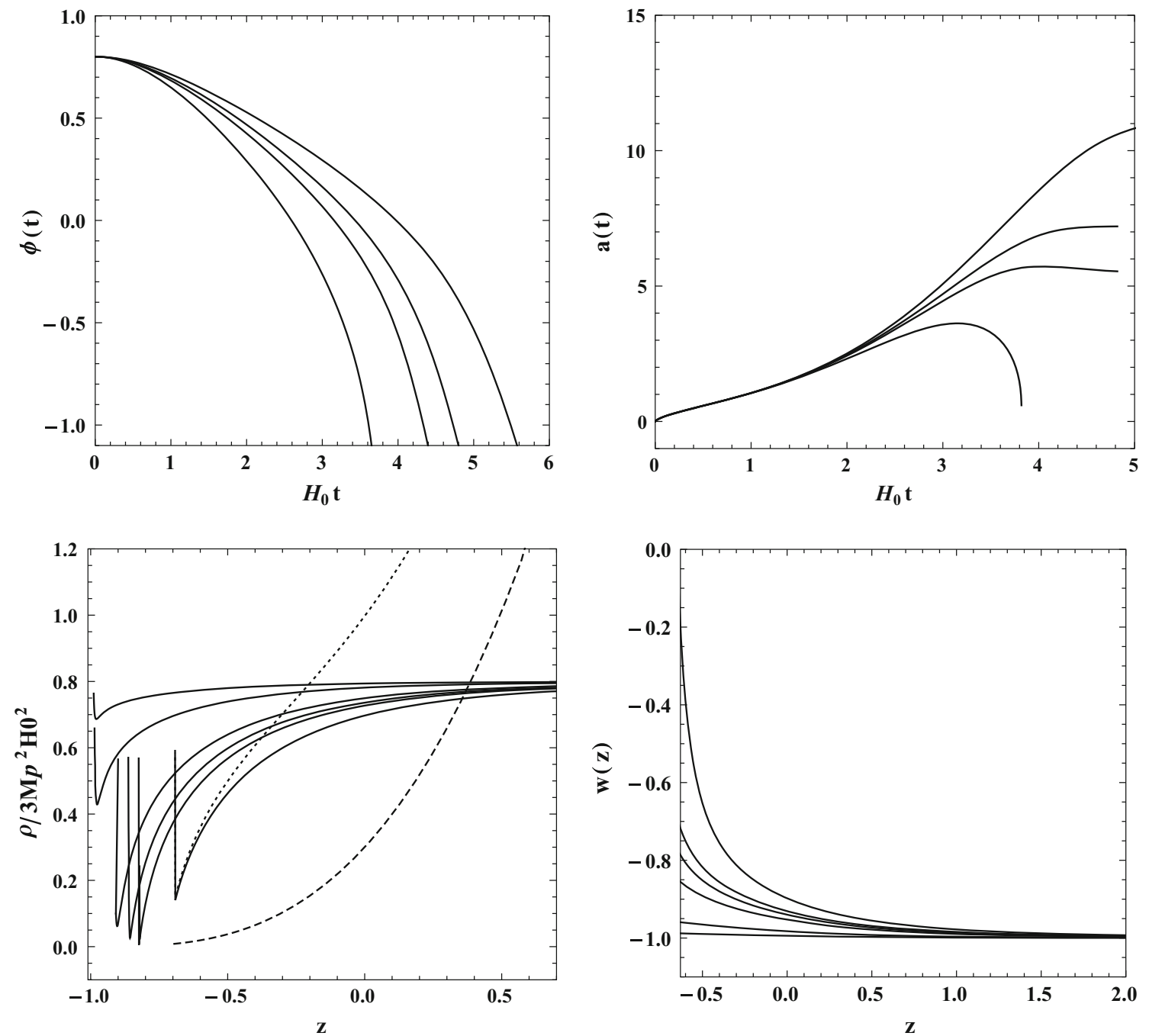

Fig. 1 The upper panels show the evolution of field $\phi(t)$ and scale factor $a(t)$ versus time $\left(H_{0} t\right)$ for a Galileon field having a linear potential for different values of $\beta$ and $V_{0}=1$ showing the collapse nature in the future (see upper right panel). The time is normalised by $H_{0}$ (Hubble constant at present epoch). The present time corresponds to $t_{0}=0.96$. The left bottom panel represents the evolution of energy density $\rho$ versus redshift $z$. The solid lines correspond to Galileon field for different values of $\beta$. The dashed and dotted lines represent the

ature. However, it is plagued with intense quantum instabilities. Theoretically, we still do not know the basic origin of $w<-1$. In the recent past, it has been discussed that the opposite sign in the kinetic energy term does not give instabilities, required that higher order derivative terms should be included in the action [97].

We take the Galileon phantom field model by invoking a negative sign in the kinetic energy term. For $\beta=0$, it behaves as a standard phantom field model. In this case, the initial kinetic term of the phantom field decreases due to the Hubble damping term in Eq. (10) and as a result the field freezes for a while till the epoch $\rho_{\phi}$ approaches $\rho_{m}$ (see bottom left panel of Fig. 2). Eventually, the field switches

energy density of matter and the total energy density of the Universe respectively with $\beta=0$ (quintessence). The right bottom panel shows the evolution of the equation of state parameter $w$ versus redshift $z$ for the Galileon field with different values of $\beta$. In this figure, upper panels are plotted for $\beta=0,0.3,0.5,1$ (for higher values, the collapse shifted to a more far distant future) whereas the lower panels have $\beta=0,0.3,0.5,1,10,100$ from bottom to top but in the bottom right panel from top to bottom

on and the future evolution depends upon the shape of the potential $V(\phi)$.

When $\dot{\phi}$ is nearly frozen and the phantom energy starts to dominate, then the system of equations (2) and (5) reduces to (for $\beta=0$ case)

$H^{2} \simeq \frac{V(\phi)}{3 M_{p l}^{2}}, \quad \dot{\phi} \simeq \frac{V^{\prime}(\phi)}{3 H}$.

The ratio of kinetic to potential terms can be written as

$\frac{\dot{\phi}^{2}}{2 V(\phi)}=\frac{M_{p l}^{2}}{6} \frac{V^{\prime 2}}{V(\phi)^{2}}=\frac{M_{p l}^{2}}{6} \frac{1}{\phi^{2}}$, 

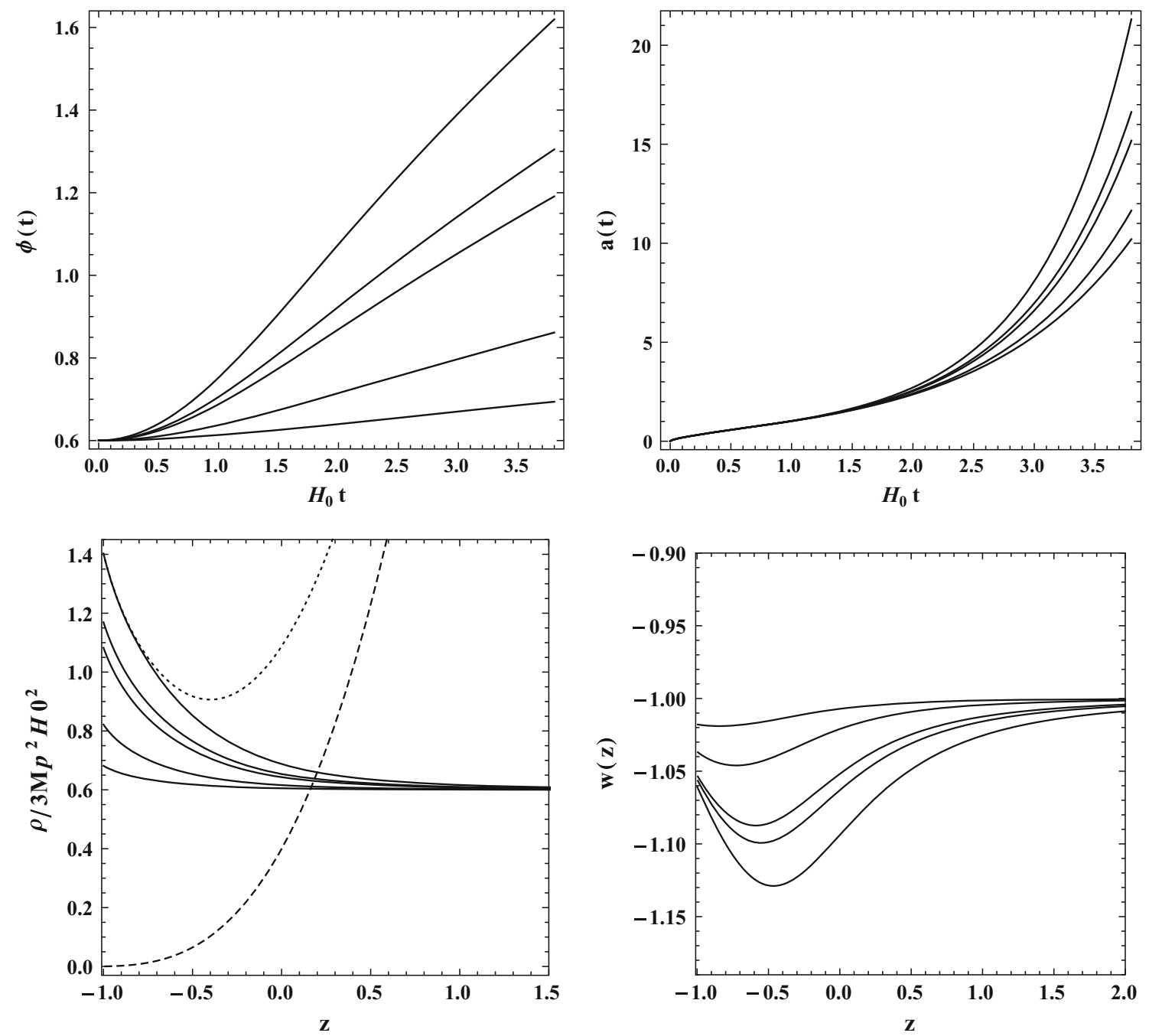

Fig. 2 The evolution of field $\phi(t)$ and scale factor $a(t)$ versus time $\left(H_{0} t\right)$ for Galileon phantom field with linear potential are plotted and shown in the upper panels for different values of $\beta$ and $V_{0}=1$. The time is normalised by $H_{0}$ and the present time is $t_{0}=0.96$. The upper right panel shows the divergent nature of the scale factor, in future, after some finite interval of time. The energy density $\rho$ versus redshift $z$ is shown in the left bottom panel, where the solid lines correspond to

where $V(\phi)=V_{0} \phi / M_{p l}$, the ratio of kinetic energy to potential energy terms is proportional to $1 / \phi^{2}$ and goes to zero; as a result the kinetic energy term remains subdominant continually. This is similar to the slow-roll regime for an ordinary field and can be called "slow climb" [45,98100]. How to exit from the rip was discussed in Ref. [99]. The equation of state approaches -1 (see bottom right panel of Fig. 2) with an increasing energy density as shown in the bottom left panel of Fig. 2. The estimation $\frac{\dot{\phi}^{2}}{2 V(\phi)} \longrightarrow 0$ is not valid for an exponential and steeper potentials. We shall discuss this case in Sect. 3. In the case of a phantom with a Galileon correction (for $\beta \neq 0$ ), with the domination of the phantom energy and $\dot{\phi}$ being small, the system of equations (2) and (5) reduces to (by taking the subleading terms)

Galileon phantom field for various values of $\beta$. The dashed and dotted lines represent the energy density of matter and the total energy density of the Universe, respectively, for $\beta=0$ (standard phantom). The right bottom panel shows the evolution of the equation of state $w$ versus redshift $z$ for the Galileon phantom field with various values of $\beta$. In this figure, all the panels have $\beta=0,0.5,1,10$ and 100 from top to bottom and in the bottom right panel from bottom to top

$H^{2} \simeq \frac{V(\phi)}{3 M_{p l}^{2}}, \quad \dot{\phi} \simeq \frac{M_{p l}^{3}}{6 \beta H}\left[-1 \pm \sqrt{1+\frac{4 \beta}{M_{p l}^{3}} V^{\prime}(\phi)}\right]$.

In the slow-roll approximation, the term $\frac{4 \beta}{M_{p l}^{3}} V^{\prime}(\phi)$ is small and we have from (17)

$\frac{\dot{\phi}^{2}}{2 V} \approx \frac{M_{p l}^{2}}{6}\left(\frac{V^{\prime}(\phi)}{V(\phi)}\right)^{2}\left[1-\frac{2 \beta}{M_{p l}^{3}} V^{\prime}(\phi)\right]$,

showing that the presence of a Galileon correction term enhances the slow climb for monotonically increasing $V(\phi)$. Keeping in mind $\epsilon=-\frac{\dot{H}}{H^{2}}=\frac{3}{2}\left(1+\omega_{e f f}(\phi)\right)$, we have shown numerically that the Galileon correction term for large 

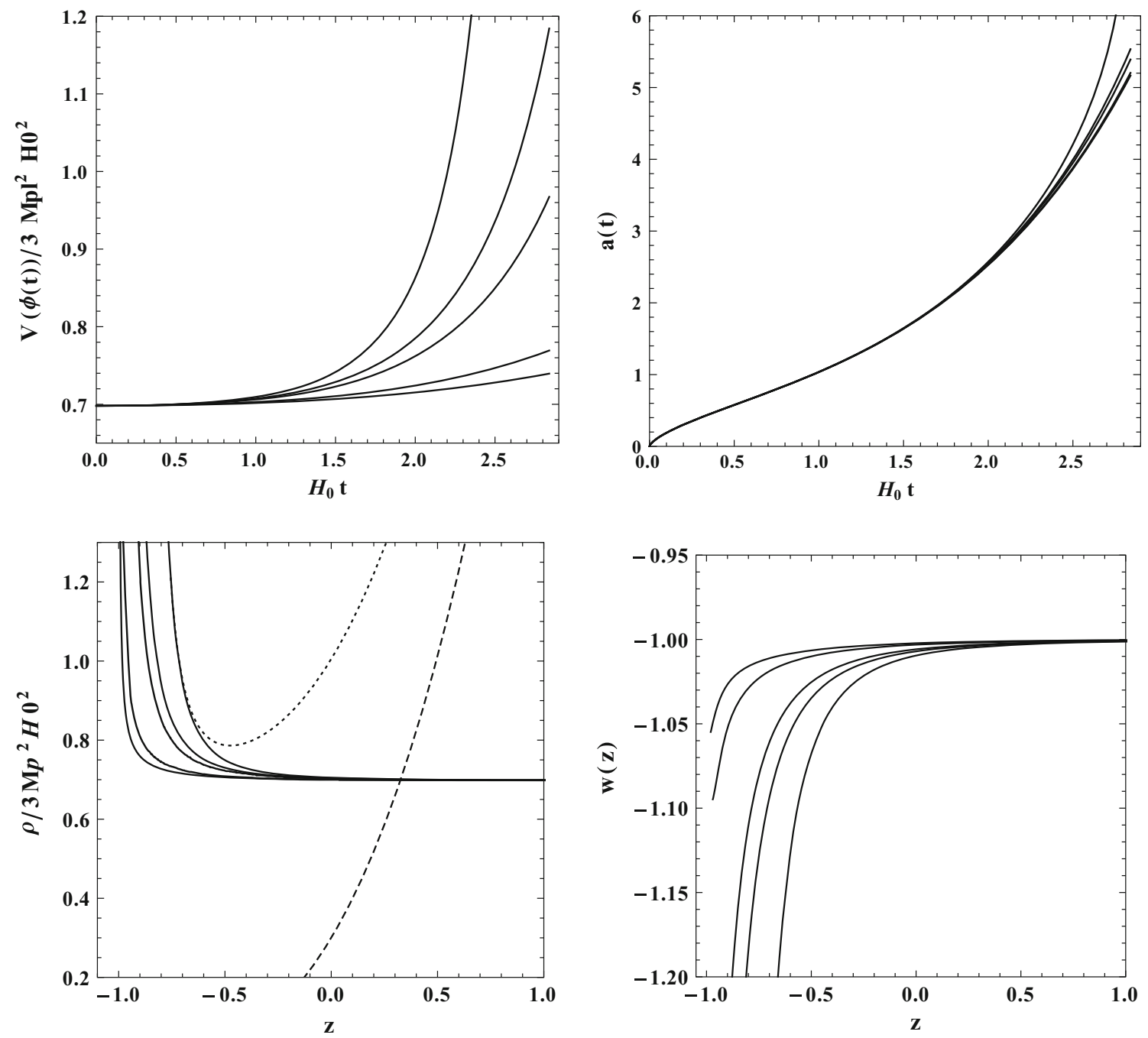

Fig. 3 The upper panels show the evolution of potential $V(\phi(t))$ and scale factor $a(t)$ versus time $\left(H_{0} t\right)$ for Galileon phantom field with exponential potential which is more steeper than the linear potential for different values of $\beta$. The scale factor $a(t)$ shows the divergent nature after a finite interval of time (in distant future). Here also the time is normalised by $H_{0}$ and the present time corresponds to $t_{0}=0.96$. The left bottom panel represents the evolution of energy density $\rho$ versus redshift $z$. The solid lines correspond to the energy density of the Galileon phantom field with exponential potential for various values of $\beta$ whereas dotted and dashed lines represent the energy density of matter and total energy density of the Universe, respectively, for standard phantom field.

values of $\beta$ moves $\omega_{e f f}(\phi)$ towards the de Sitter point, though $\omega_{\text {eff }}(\phi)$ yet remains less than -1 .

In the upper panels of Fig. 2, we show the evolution of the phantom field $\phi$ and the scale factor $a$ versus time. The phantom field and the scale factor both diverge after a finite interval of time (in the future) and correspondingly the energy density of the phantom field $\rho_{\phi}$ increases slowly (see bottom left panel of Fig. 2). In this case, the equation of state first decreases from -1 and then eventually increases towards

At late times, the energy density of the field gets to the matter, overtakes it and begins to increase $(w<-1)$, and acquires the present accelerated expansion of the Universe. Afterwards $\rho_{\phi}$ continuously blows up, in the future, after a finite interval of time. The right bottom panel shows the evolution of the equation of state $w$ versus redshift $z$ for a Galileon phantom field with steep exponential potential. It has another type of future singularity than the less steeper potential (linear potential) and continuously blows up to $-\infty$ after definite interval of the redshift. For larger values of $\beta$, the Big Rip singularity is delayed in distant future. In this figure, all the plots have $V_{0}=1.2$ and $\beta=0,0.5,1,5,10$ from top to bottom and for the bottom right plot from bottom to top

-1 and comes close to it asymptotically [45] as is shown in the bottom right panel of Fig. 2. As to this effect, the final Universe would be different from both the de Sitter phase and the Big Rip, and an infinite time would be taken to reach an infinite energy density.

When we add a Galileon correction term and go to higher values of $\beta(0.5,1,10,100)$, the scale factor shows a less divergent nature than the case of $\beta=0$ and correspondingly $\rho_{\phi}$ grows slowly as shown in the upper right and left bottom 
panels of Fig. 2; initially, the Galileon phantom field imitates the $\Lambda \mathrm{CDM}$ like behaviour and its energy density is highly sub-dominant to the matter energy density $\rho_{m}$ and remains so, for most of the time of evolution. The Galileon phantom field remains in the state with $w=-1$ till the epoch $\rho_{\phi}$ goes near to $\rho_{m}$. At late times, the energy density of the Galileon phantom field approaches matter, overtakes it and begins to increase $(w<-1)$, and acquires the present accelerated expansion of the Universe having $\Omega_{0 m} \simeq 0.3$ and $\Omega_{0 \phi} \simeq 0.7$. For higher values of $\beta(\beta=0.5,1,10,100)$ the slow growing divergence shifted towards lower values of $\rho_{\phi}$. In the right bottom panel of Fig. 2, we present the evolution of $w$ versus redshift $z$ for Galileon phantom field. For $\beta=0$, the equation of state of Galileon phantom field deviates more from the equation of state of the $\Lambda \mathrm{CDM}$ model. As the values of $\beta$ are increased, we get less deviation from $\Lambda \mathrm{CDM}$. For all values of $\beta$, the equation of state first decreases from -1 and then subsequently increases towards -1 and comes near to it asymptotically. Hence, we get smaller deviation in equation of state parameter $w$ from $\Lambda \mathrm{CDM}$ for $\beta>0$. The effect of the Galileon correction on the evolution of the phantom field was also studied in Ref. [100].

\section{Galileon phantom field with exponential potential}

We consider the Galileon phantom field with exponential potential. This is a purely phenomenological case that is just to establish more liberty and workability. However, this type of potential breaks the Galileon shift symmetry. It is like most of the phantom field models in which potentials are completely phenomenological. The system of equations (4) and (5) with Eq. (8) for an exponential potential can be written as

$$
\begin{aligned}
\frac{\ddot{a}}{a} & \dot{\phi}^{2}+\frac{3 \sqrt{3} \beta}{2}\left(\frac{H_{0}}{M_{p l}}\right)^{2}\left(\frac{\dot{a}}{a} \dot{\phi}^{3}-\dot{\phi}^{2} \ddot{\phi}\right)+\frac{V_{0}}{\sqrt{3}} e^{3 \phi^{2}} \\
& -\frac{\Omega_{0 m}}{2 a^{3}}, \\
\ddot{\phi}+ & 3 \frac{\dot{a}}{a} \dot{\phi}+3 \sqrt{3} \beta \dot{\phi}\left(\frac{H_{0}}{M_{p l}}\right)^{2}\left\{3 \frac{\dot{a}^{2}}{a^{2}} \dot{\phi}+\left(\frac{a \ddot{a}-\dot{a}^{2}}{a^{2}}\right) \dot{\phi}\right. \\
& \left.+2 \frac{\dot{a}}{a} \ddot{\phi}\right\}=2 \sqrt{3} V_{0} \phi e^{3 \phi^{2}},
\end{aligned}
$$

where we have used $V(\phi)=V_{0} \exp \left(\phi^{2} / M_{p l}^{2}\right)$. Now, we solve the system of equations (19) and (20) numerically with Eq. (12).

For the case of $\beta=0$, the Galileon phantom field model becomes the standard phantom field model. Here we consider an exponential potential which is steeper than the linear one. In this potential, we obtain a different type of future singularity. The scale factor $a(t)$ diverges in the distant future after a finite interval of time as shown in the upper right panel of Fig. 3. In exponential potentials, Hao and Li obtained an attractor solution having $w<-1$ rendering the "Big Rip" unavoidable [101]. Our numerical analysis shows that the energy density of the phantom field blows up after a finite interval of time and correspondingly the equation of state parameter $w$ blows up to $-\infty$ (see bottom panels of Fig. 3). This type of singularity has been discussed in different models, namely, brane worlds [102], Gauss-Bonnet cosmology [103] and tachyonic field [104]. For higher values of $\beta$, the Big Rip singularity is shifted into the distant future. One can say that the Big Rip singularity is delayed for $\beta>0$.

\section{Conclusion}

In this paper, we have investigated cosmological dynamics of the phantom and non-phantom fields in the presence of a higher derivative Galileon correction $\mathcal{L}_{3}$. For generality, we also studied the phantom field with a general potential in order to check the impact of the Galileon term on the structure of the singularity.

In the case of $\beta=0$, the Galileon field (with a linear potential) reduces to standard quintessence. In this case, as the field evolves to the region of negative values of the potential, after a finite interval of time (in the future), the scale factor collapses, giving rise to a Big Crunch singularity. To this effect, the energy density of the Galileon field $\rho_{\phi}$ shows the collapse nature in the future. In the case of the standard Galileon field with a linear potential, the Big Crunch singularity can be delayed, depending upon the numerical values of $\beta$ such that for large values of the parameter, the delay may be considerable, making the singularity practically redundant (see Fig. 1).

As for the phantom field, there are three types of singularities depending upon the nature of potential. In the case of $V \sim \phi^{n}$, the energy density diverges after an infinite time for $n \leq 4$, whereas divergence is reached after finite time dubbed Big Rip if $n>4$ including the case of an exponential potential, which corresponds to $n \rightarrow \infty$. In the case of potentials steeper than the standard exponential, not only divergence of the scale factor is reached in a finite time but the equation of state parameter also diverges accordingly.

We have examined the probable future regimes of Universe with Galileon phantom field having a linear potential. In the case of $\beta=0$, the Galileon phantom field reduces to the standard phantom. Due to the negative sign in the kinetic term, the field increases along with the potential, giving rise to a singularity in the future. The nature of this singularity is different for different types of potentials. In the case of a linear potential with a Galileon phantom field, the equation of state parameter $w$ approaches -1 with the slowly growing energy density compared to the standard case. For various 
values of $\beta(0,0.5,1,10,100)$, we display our results in Fig. 2, which shows that the equation of state has less and less deviation from $\Lambda$ CDM for larger values of $\beta$ and asymptotically approaches -1 in the distant future with the slowly increasing energy density as shown in the bottom panels of Fig. 2. In the case of an exponential potential, which is steeper than the linear one, it has a different type of singularity, the equation of state blows up to $-\infty$ for a definite value of the redshift and correspondingly the energy density $\rho_{\phi}$ diverges (see the bottom panels of Fig. 3); that is, during a definite time an infinite energy density is reached and this is termed the "Big Rip" singularity which will rip galaxies apart some billion years before the actual Rip singularity is reached [105]. In this case, the larger values of $\beta$ will delay the Big Rip singularity towards the more and more distant future. We therefore conclude that in general the effect of a Galileon correction to the standard kinetic term in the Lagrangian generally results in a delayed approach of the singularity. It might be interesting to investigate the behaviour of the singularity using the full Galileon Lagrangian including $\mathcal{L}_{4}$ and $\mathcal{L}_{5}$. Again, apart from the Big Crunch or Big Rip singularities, it will be more interesting to study the effect of a Galileon correction term on the other singularities like the pressure singularity or sudden singularity [106] and the softer type-IV singularity [107], which have extensively been studied in [108] and [109]; all this is postponed to future investigations.

Acknowledgments We thank M. Sami for his useful comments and suggestions. MS acknowledges the financial support provided by the University Grants Commission, Government of India, under the scheme of Dr. D. S. Kothari Post-doctoral Fellowship. He is also thankful to M. Sajjad Athar for his constant encouragement throughout the work. The author SKJP wish to thank Department of Atomic Energy (DAE), Government of India for financial support through the post-doctoral fellowship of National board of Higher Mathematics (NBHM).

Open Access This article is distributed under the terms of the Creative Commons Attribution 4.0 International License (http://creativecomm ons.org/licenses/by/4.0/), which permits unrestricted use, distribution, and reproduction in any medium, provided you give appropriate credit to the original author(s) and the source, provide a link to the Creative Commons license, and indicate if changes were made. Funded by SCOAP ${ }^{3}$.

\section{References}

1. S. Perlmutter et al., Astrophys. J. 517, 565 (1999)

2. A.G. Reiss et al., Astron. J. 116, 1009 (1998)

3. N.A. Bachall et al., Science 284, 1481 (1999)

4. A.G. Reiss et al., Astrophys. J. 607, 665 (2004)

5. R.A. Knop et al., Astrophys. J. 517, 565 (1999)

6. E.J. Copeland, M. Sami, S. Tsujikawa, Int. J. Mod. Phys. D 15, $1753(2006)$

7. V. Sahni, A.A. Starobinsky, Int. J. Mod. Phys. D 9, 373 (2000)

8. B. Ratra, P.J.E. Peebles, Phys. Rev. D 37, 3406 (1988)

9. C. Wetterich, Nucl. Phys. B 302, 668 (1988)
10. R.R. Caldwell, R. Dave, P.J. Steinhardt, Phys. Rev. Lett. 80, 1582 (1998)

11. I. Zlatev, L. Wang, P.J. Steinhardt, Phys. Rev. Lett. 82, 896 (1999)

12. V. Sahni, M. Sami, T. Souradeep, Phys. Rev. D 65, 023518 (2002)

13. M. Sami, N. Dadhich, T. Shiromizu, Phys. Lett. B 568, 118 (2003)

14. M. Sami, T. Padmanabhan, Phys. Rev. D 67, 083509 (2003)

15. C. Armendariz-Picon, T. Damour, V. Mukhanov, Phys. Lett. B 458, 209 (1999)

16. T. Chiba, T. Okabe, M. Yamaguchi, Phys. Rev. D 62, 023511 (2000)

17. L.A. Boyle, R.R. Caldwell, M. Kamionkowski, Phys. Lett. B 545, 17 (2002)

18. A. Sen, J. High Energy Phys. 0207, 065 (2002)

19. T. Padmanabhan, Phys. Rev. D 66, 021301 (2002)

20. E. Elizalde, S. Nojiri, S.D. Odintsov, Phys. Rev. D 70, 043539 (2004). arXiv:hep-th/0405034

21. B. Feng, X.L. Wang, X.M. Zhang, Phys. Lett. B 607, 35 (2005)

22. Z.K. Guo, Y.S. Pio, Y.Z. Zhang, X.M. Zhang, Phys. Lett. B 608, 177 (2005)

23. A. Anisimov, E. Babichev, A. Vikman, J. Cosmol. Astropart. Phys. 0506, 006 (2005)

24. M.R. Setare, J. Sadeghi, A.R. Amani, Phys. Lett. B 660, 299 (2008)

25. J. Sadeghi, M.R. Setare, A. Banijamali, F. Milani, Phys. Lett. B 662, 92 (2008)

26. M.R. Setare, E.N. Saridakis, Phys. Lett. B 668, 177 (2008)

27. M.R. Setare, E.N. Saridakis, J. Cosmol. Astropart. Phys. 09, 026 (2008)

28. J. Khoury, A. Weltman, Phys. Rev. Lett. 93, 171104 (2004)

29. J. Khoury, A. Weltman, Phys. Rev. D 69, 044026 (2004)

30. P. Brax et al., Phys. Rev. D 70, 123518 (2004)

31. A. Sattar, S.R. Prajapati, Int. J. Theor. Phys. 50, 2355 (2011)

32. L. Parker, A. Raval, Phys. Rev. D 60, 063512 (1999)

33. V. Sahni, A. Starobinsky, Int. J. Mod. Phys. D 9, 373 (2000)

34. B. Boisseau, G. Esposito-Farese, D. Polarski, A.A. Starobinsky, Phys. Rev. Lett. 85, 2236 (2000)

35. A.E. Schulz, M. White, Phys. Rev. D 64, 043514 (2001)

36. R.R. Caldwell, Phys. Lett. B 545, 23 (2002)

37. V. Faraoni, Int. J. Mod. Phys. D 64, 043514 (2002)

38. I. Maor, R. Brustein, J. Mcmahon, P.J. Steinhardt, Phys. Rev. D 65, 123003 (2002)

39. V.K. Onemli, R.P. Woodard, Class. Quant. Grav. 19, 4607 (2002)

40. D.F. Torres, Phys. Rev. D 66, 043522 (2002)

41. S. Hannestad, E. Mortsell, Phys. Rev. D 66, 063508 (2002)

42. P. Singh, M. Sami, N. Dadhich, Phys. Rev. D 68, 023522 (2003)

43. G.W. Gibbons, Phantom matter and the cosmological constant. arXiv:hep-th/302199

44. S. Nojiri, S.D. Odintsov, Phys. Lett. B 562, 147-152 (2003). arXiv:hep-th/0303117

45. M. Sami, A. Toporensky, Mod. Phys. Lett. A 19, 1509 (2004)

46. V. Sahni, Yu Shtanov. arXiv:astro-ph/0202346

47. V. Faraoni. arXiv:gr-qc/0307086

48. M. Sami, M. Shahalam, M. Skugoreva, A. Toporensky, Phys. Rev. D 86, 103532 (2012)

49. M. Shahalam, S. Sami, A. Agarwal, Mon. Not. R. Astron. Soc. 448, 2948-2959 (2015) arXiv:1501.04047

50. B. McInnes. arXiv:hep-th/0305107

51. M.N.R. Wohlfarth. arXiv:hep-th/0307179

52. N. Ohta. arXiv:hep-th/0303238

53. N. Ohta. arXiv:hep-th/0304172

54. S. Roy. arXiv:hep-th/0304084

55. M. Gutperle, R. Kallosh, A. Linde. arXiv:hep-th/0304225

56. C.-M. Chen, P.-M. Ho, I.P. Neupane, J.E. Wang. arXiv:hep-th/0304177

57. C.-M. Chen, P.-M. Ho, I.P. Neupane, N. Ohta, J.E. Wang. arXiv:hep-th/0306291 
58. I.P. Neupane. arXiv:hep-th/0311071

59. F. Hoyle, J.V. Narlikar, Proc. Roy. Soc. A282, 191 (1964)

60. F. Hoyle, J.V. Narlikar, Mon. Not. R. Astr. Soc. 155, 305 (1972)

61. F. Hoyle, J.V. Narlikar, Proc. Roy. Soc. 155, 323 (1972)

62. A. Starobinsky, Grav. Cosmol. 6, 157 (2000). arXiv:astro-ph/9912054

63. S.M. Carroll, M. Hoffman, M. Trodden. arXiv:astro-ph/0301273

64. V.K. Onemli, R.P. Woodard, Class. Quant. Grav. 19, 4607 (2002). arXiv:gr-qc/0204065

65. V.K. Onemli, R.P. Woodard, Phys. Rev. D 70, 107301 (2004). arXiv:gr-qc/0406098

66. A. Nicolis, R. Rattazzi, E. Trincherini, Phys. Rev. D 79, 064036 (2009)

67. A. Nicolis, R. Rattazzi, JHEP 06, 059 (2004)

68. A. De Felice, S. Tsujikawa, Phys. Rev. Lett. 105, 111301 (2010)

69. S. Appleby, E.V. Linder, JCAP 03, 043 (2012)

70. M. Jamil, D. Momeni, R. Myrzakulov, Eur. Phys. J. C 73, 2347 (2013)

71. A. De Felice, S. Tsujikawa, JCAP 03, 025 (2012)

72. A. De Felice, R. Kase, S. Tsujikawa, Phys. Rev. D 83, 043515 (2011)

73. S. Nesseris, A. De Felice, S. Tsujikawa, Phys. Rev. D 82, 124054 (2010)

74. C. Burrage, C. de Rham, D. Seery, A.J. Tolley, JCAP 01, 014 (2011)

75. A. De Felice, S. Tsujikawa, Phys. Rev. D 84, 124029 (2011)

76. S. Bhattacharya, P. Mukherjee, A. Singha Roy, A. Saha. arXiv: 1512.03902

77. D. Momeni, M.J.S. Houndjo, E.GÃ $\frac{1}{4}$ dekli, M.E. Rodrigues, F.G. Alvarenga, R. Myrzakulov, Int. J. Theor. Phys. 55(2), 12111221 (2016). arXiv:1412.4672 [bibgr-qc]

78. A. Ali, R. Gannouji, M. Sami, Phys. Rev. D 82, 103015 (2010)

79. R. Gannouji, M. Sami, Phys. Rev. D 82, 024011 (2010)

80. G.R. Dvali, G. Gabaddze, M. Porrati, Phys. Lett. B 485, 208 (2000)

81. M.A. Luty, M. Porrati, R. Rattazzi, JHEP 09, 029 (2003)

82. A. Ali, R. Gannouji, M.W. Hossain, M. Sami, Phys. Lett. B 718 , 5 (2012)

83. M.W. Hossain, A.A. Sen, Phys. Lett. B. 713, 140 (2012)

84. R. Myrzakulov, M. Shahalam, Gen. Rel. Grav. 47, 81 (2015)

85. R. Kallosh, J. Kratochvil, A.D. Linde, E.V. Linder, M. Shmakova, JCAP 0310, 015 (2003). arXiv:astro-ph/0307185 [astro-ph]

86. J. Garriga, L. Pogosian, T. Vachaspati, Phys. Rev. D 69, 063511 (2004). arXiv:astro-ph/0311412 [astro-ph]
87. L. Perivolaropoulos, Phys. Rev. D 71, 063503 (2005). arXiv:astro-ph/0412308 [astro-ph]

88. Y. Wang, J.M. Kratochvil, A.D. Linde, M. Shmakova, JCAP 0412, 006 (2004). arXiv:astro-ph/0409264 [astro-ph]

89. R.Z. Ferreira, P.P. Avelino. arXiv:1508.00631v1 [astro-ph.co]

90. A. Lykkas, L. Perivolaropoulos. arXiv:1511.08732v1 [gr-qc]

91. R.J. Scherrer, A.A. Sen, Phys. Rev. D 77, 083515 (2008)

92. R.J. Scherrer, A.A. Sen, Phys. Rev. D 78, 067303 (2008)

93. S. Sen, A.A. Sen, M. Sami, Phys. Lett. B. 686, 1 (2010)

94. S. del Campo, C.R. Fadragas, R. Herrera, C. Leiva, G. Leon, J. Saavedra, Phys. Rev. D 88, 023532 (2013). arXiv:1303.5779

95. D. Escobar, C.R. Fadragas, G. Leon, Y. Leyva, Astrophys. Space Sci. 349, 575 (2014). arXiv:1301.2570

96. P.A.R. Ade et al. Planck Collaboration. arXiv:1303.5076 [astroph.CO]

97. N. Arkani-Hamed, H.C. Cheng, M.A. Luty, S. Mukohyama, 2003 Preprint. arXiv:hep-th/0312099

98. Y.-S. Piao, Y.-Z. Zhang, Phys. Rev. D 70, 063513 (2004) arXiv:astro-ph/0401231

99. Z.-G. Liu, Y.-S. Piao, Phys. Lett. B 713, 53-58 (2012). arXiv:1203.4901

100. Z.-G. Liu, J. Zhang, Y.-S. Piao, Phys. Rev. D 84, 063508 (2011). arXiv: 1105.5713

101. J.-G. Hao, X.-Z. Li, Phys. Rev. D 69, 107303 (2004). arXiv:hep-th/0303093

102. Yu. Shtanov, V. Sahni, Class. Quant. Grav. 19, L101-L107 (2002). arXiv:gr-qc/0204040

103. A. Toporensky, S. Tsujikawa, Phys. Rev. D 65, 123509 (2002). arXiv:gr-qc/0202067

104. V. Gorini, AYu. Kamenshchik, U. Moschella, V. Pasquier. Phys. Rev. D 69, 123512 (2004). arXiv:hep-th/0311111

105. R.R. Caldwell, M. Kamionkowski, N.N. Weinberg, Phys. Rev. Lett. 91, 071301 (2003). arXiv:astro-ph/0302506

106. J.D. Barrow, Class. Quant. Grav. 21, L79-L82 (2004). arXiv:gr-qc/0403084

107. S.D. Odintsov, V. K. Oikonomou, Phys. Rev. D 92 no.12, 124024 (2015). arXiv:1510.04333 [gr-qc]

108. S.D. Odintsov, V.K. Oikonomou, Phys. Rev. D 92 no.2, 024058 (2015). arXiv:1507.05273 [gr-qc]

109. S. Nojiri, S.D. Odintsov, V.K. Oikonomou, E.N. Saridakis, JCAP 1509, 044 (2015). arXiv:1503.08443 [gr-qc] 